\title{
Gramíneas nativas potenciais para revegetação de áreas degradadas, a partir da avaliação de topsoil de Campo Rupestre Ferruginoso ${ }^{1}$
}

\author{
Diogo Felcar Saraiva ${ }^{2,4}$, (D) Soraya Alvarenga Botelho², (D) Cláudio Coelho de Paula ${ }^{3,4}$, (D) Ronaldo Vinícius-Silva ${ }^{4,6}$, \\ Paulo José de Morais ${ }^{5}$ e (D) Lucas de Amaral de Melo $^{2}$
}

Recebido: 26.12.2019; aceito: 16.06 .2020

Como citar: Saraiva, D.F., Botelho, S.A., Paula, C.C., Vinícius-Silva, R., Morais, P.J. \& Melo, L.A. 2020. Gramíneas nativas potenciais para revegetação de áreas degradadas, a partir da avaliação de topsoil de Campo Rupestre Ferruginoso. Hoehnea 47: e1122019. http://dx.doi.org/10.1590/2236-8906-112/2019

\begin{abstract}
Native Brazilian grass species with potential for revegetation of degraded lands, surveyed from a Ferruginous Campo Rupestre topsoil). An alternative to find adequate species to revegetate mined sites is surveying the species that develop from the local topsoil seed bank. In that sense, grass species are usually the most indicated ones, as they contribute to stabilizing soil. We aimed to evaluate the native species that would develop from the seed bank of a ferruginous topsoil and select the grass species with highest importance value indexes (IVI's) for their potential of use in revegetation programs. The evaluated topsoil was deposited in the Gerdau's Unit for Research and Innovation on Ferruginous Rocky Outcrops at Ouro Branco, Minas Gerais State, Brazil, where phytosociological studies were conducted upon seed bank development. Eragrostis polytricha Nees showed highest values not only of IVI but also of relative frequency and relative cover, in addition to the second highest value of relative density. Thus, we recommend E. polytricha as a potential species for being used in revegetation programs. Keywords: Eragrostis polytricha, mining activity, native grass species, revegetation
\end{abstract}

RESUMO - (Gramíneas nativas potenciais para revegetação de áreas degradadas, a partir da avaliação de topsoil de Campo Rupestre Ferruginoso). Uma das alternativas para se obter espécies potenciais para a revegetação de áreas degradadas é utilizar as que se desenvolvem a partir do banco de sementes de topsoil e nesse contexto, as gramíneas são as mais indicadas por possuírem características associadas à estabilização do solo. Este estudo objetivou avaliar as espécies nativas desenvolvidas em banco de sementes de topsoil ferruginoso e selecionar as gramíneas de maior índice de valor de importância (IVI), considerando-as espécies em potencial para utilização em programas de revegetação. O topsoil foi depositado na Unidade de Pesquisa e Inovação em Campos Rupestres Ferruginosos da Gerdau, Ouro Branco, MG, Brasil e após a germinação e estabelecimento das plantas, realizou-se estudos fitossociológicos. Eragrostis polytricha Nees apresentou maiores valores não apenas de IVI, mas também de Frequência Relativa e Cobertura Relativa, adicionalmente apresentou o segundo maior valor de Densidade Relativa. Portanto, recomendamos E. polytricha para a utilização em programas de revegetação. Palavras-chave: atividade mineradora, Eragrostis polytricha, gramíneas nativas, revegetação

\section{Introdução}

A mineração, em um âmbito geral, acompanha a evolução humana e tem desempenhado um papel importante tanto do ponto de vista econômico quanto social, sendo, no Brasil, responsável por um considerável aumento no PIB (Produto
Interno Bruto) e por geração de empregos diretos (IBRAM 2015). No entanto, essa atividade promove uma alteração nas condições ambientais, modificando a paisagem e ocasionando degradação de extensas áreas a qual atinge a vegetação, solo e água (Araújo et al. 2005). Diante disso, torna-se fundamental estratégias que visem a restauração desses locais, recuperando

1. Parte da Dissertação de Mestrado do primeiro Autor

2. Universidade Federal de Lavras, Departamento de Ciências Florestais, Campus Universitário, 37200-000 Lavras, MG, Brasil

3. Universidade Federal de Viçosa, Departamento de Biologia Vegetal, Avenida P.H. Rolfs s.n., Campus Universitário, 36570-900 Viçosa, MG, Brasil

4. Unidade de Pesquisa e Inovação em Campos Rupestres Ferruginosos da Gerdau, Fazenda do Cadete s.n., Rodovia MG-443, Km 07, 36420-000 Ouro Branco, MG, Brasil

5. Universidade Federal de Viçosa, Departamento de Fitotecnia, Avenida P.H. Rolfs s.n., Campus Universitário, 36570-900 Viçosa, MG, Brasil

6. Autor para correspondência: ronaldovinybio@yahoo.com.br 
processos e funções ecossistêmicas. Para isso, um importante passo é a revegetação da área com um compromisso de reestabelecer a estrutura vertical com as principais famílias e gêneros da vegetação anterior (Salomão 2015).

No cenário nacional, a explotação de ferro é uma das principais atividades do setor minerário (IBRAM 2015, DNPM 2016) e os Campos Rupestres Ferruginosos, designados também como vegetação de canga, são ecossistemas normalmente associados aos depósitos de minério de ferro e por isso intensamente impactados pela ação mineradora (Jacobi $\&$ Carmo 2008). Essas áreas detêm uma grande diversidade de espécies vegetais com elevado endemismo, porém estão entre as regiões mais ameaçadas e menos estudadas na atualidade (Jacobi et al. 2007, Jacobi \& Carmo 2008, Mendonça 2013). No Brasil, as duas principais regiões onde os Campos Rupestres Ferruginosos podem ser encontrados são a Serra de Carajás no Estado do Pará e o Quadrilátero Ferrífero (QF) no Estado de Minas Gerais (Silva et al. 1996).

Dentre as espécies potenciais para a revegetação de áreas degradadas, as gramíneas têm se destacado pois promovem a completa e perene cobertura do solo, fornecendo proteção a este de maneira eficiente (Gylssels \& Poesen 2003, Bindle 2003). Além disso, as gramíneas possuem sistema radicular denso na camada superficial do solo, o que the confere estabilidade (Gylssels \& Poesen 2003). No Brasil, a maioria dos estudos que visam a revegetação de áreas são conduzidos com gramíneas exóticas (Martins 2001). Essas espécies podem gerar graves problemas à biodiversidade local devido a sua agressividade, sendo consideradas, em geral, competitivamente superiores às nativas (Marques 2011).

Uma das estratégias para ampliar o conhecimento das espécies nativas é estudar as comunidades colonizadoras do topsoil, o qual é originado da raspagem superficial do solo e atua como um reservatório de diversidade florística e genética, podendo ser utilizado para restaurar áreas degradadas ou recuperar espécies da flora (Pérez \& Santiago 2001). A utilização do topsoil pode auxiliar na manutenção do banco de sementes local, o que condiz com uma maior semelhança em relação à área natural que foi perturbada (Rezende 2010).

Nesse sentido, utilizando-se da análise de parâmetros fitossociológicos (densidade, frequência, cobertura e índice de valor de importância) da comunidade que se estabeleceu a partir da germinação do banco de sementes do topsoil é possível inferir e selecionar espécies nativas potenciais para serem utilizadas em programas de revegetação de taludes. Diante do exposto, objetiva-se com este estudo avaliar as espécies nativas que se desenvolverem a partir do banco de sementes de topsoil proveniente de Campo Rupestre Ferruginoso e selecionar as gramíneas de maior índice de valor de importância, considerando-as como potencial para utilização em programas de revegetação.

\section{Material e Métodos}

Coleta e acondicionamento do topsoil - $\mathrm{O}$ topsoil utilizado foi obtido na preparação de uma área para a explotação de minério de ferro de uma mina localizada no extremo oeste do Quadrilátero Ferrífero (QF), 20¹7'12.88”'S, 4356'50.86”'W, na Serra da Moeda, município de Itabirito, MG, Brasil, onde foi escavado $15 \mathrm{~cm}$ de solo. Posteriormente, este foi imediatamente transportado e depositado na Unidade de Pesquisa e Inovação em Campos Rupestres Ferruginosos da Gerdau, localizada no município de Ouro Branco, MG

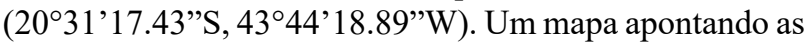
duas áreas pode ser observado na figura 1 .

O referido material é composto basicamente por substrato ferruginoso, banco de sementes, além de micro, meso e macrofauna do solo. No total foram coletadas 175 toneladas de topsoil, sendo o procedimento efetuado por meio de raspagem e conduzido com auxílio de um trator de esteira. $\mathrm{O}$ acondicionamento foi realizado em novembro de 2015 na Unidade de Pesquisa e Inovação em Campos Rupestres Ferruginosos da Gerdau (figura 2) onde foi distribuído uniformemente em uma área de $330 \mathrm{~m}^{2}$ com uma camada de aproximadamente $15 \mathrm{~cm}$ de espessura.

Coleta de dados e Análise Fitossociológica - Após um ano, a contar do período de acondicionamento do topsoil e sem nenhuma interferência ou trato cultural, as espécies se estabeleceram em função da germinação do banco de sementes. Foram alocadas um total de 168 parcelas de $1 \times$ $1 \mathrm{~m}$. As parcelas externas foram excluídas para diminuir as possíveis interferências da vegetação das áreas adjacentes e para cada $4 \mathrm{~m}^{2}$ sorteou-se a parcela a ser estudada, resultando um total de 29 parcelas (figura 3). A estrutura da vegetação foi avaliada pela escala de valor de cobertura e abundância proposta por Braun-Blanquet (1979). Em cada parcela todos os indivíduos foram avaliados, sendo que estes foram contabilizados separadamente por espécie. Para as espécies que apresentaram crescimento cespitoso, cada touceira foi considerada um indivíduo.

A suficiência amostral foi avaliada a partir da curva do coletor, representada pelo gráfico que relaciona o esforço amostral (número de indivíduos amostrados ou área amostral) cumulativo (eixo $\mathrm{X}$ ) com o número cumulativo de espécies amostradas (eixo Y) (Martins \& Santos 1999). O índice de valor de importância (IVI) de cada espécie foi calculado pela soma de sua densidade relativa (DR), frequência relativa (FR) e cobertura relativa (CR) (Braun-Blanquet 1979).

Dentre as espécies amostradas, a maioria foi identificada em campo ou com o auxílio de literatura especializada. A grafia dos binômios específicos e seus respectivos autores estão de acordo com o banco de dados "International Plant Names Index" (www.ipni.org) e a elaboração da lista de espécies segue o sistema de Classificação APG IV (2016).

\section{Resultados}

As parcelas analisadas apresentaram suficiência amostral, conforme demonstrado pela curva do coletor e o incremento de novas espécies foi estabilizado na $23^{\mathrm{a}}$ parcela (figura 4). No presente estudo foram identificados 430 indivíduos pertencentes a 42 espécies, sendo estas distribuídas em 


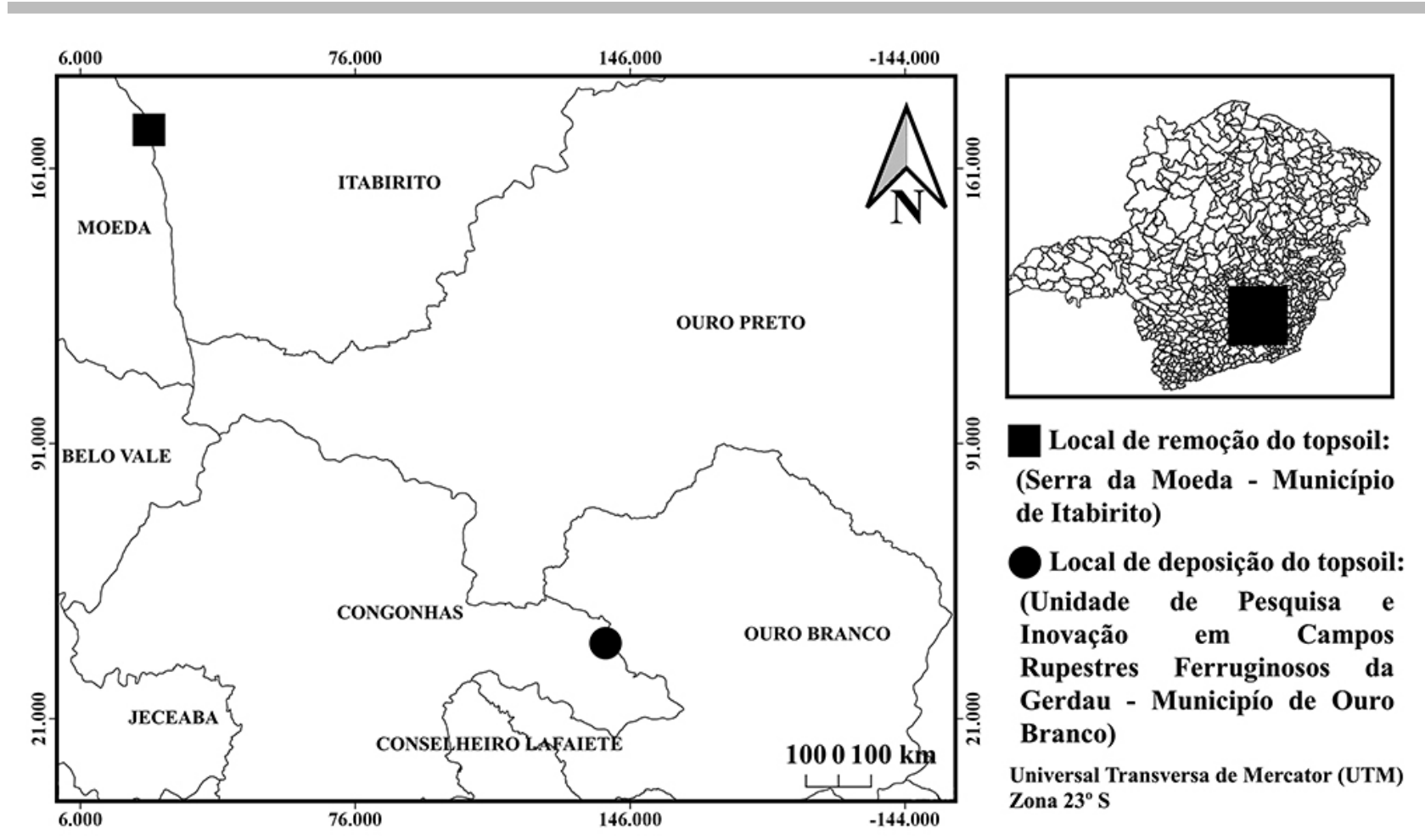

Figura 1. Mapa de distribuição com os locais de remoção e acondicionamento do topsoil, Estado de Minas Gerais, Brasil.

Figure 1. Distribution map with locations of removal and storage of topsoil, Minas Gerais State, Brazil.

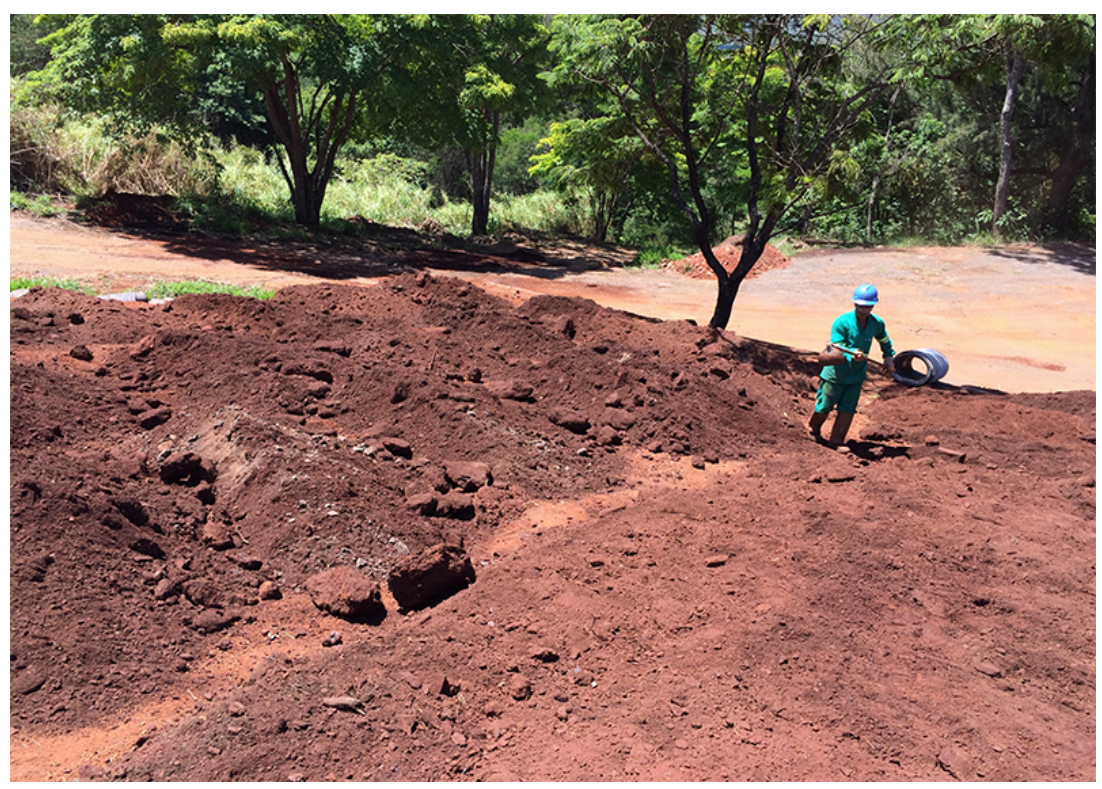

Figura 2. Área onde foi acondicionado o topsoil na Unidade de Pesquisa e Inovação em Campos Rupestres Ferruginosos da Gerdau, município de Ouro Branco, MG, Brasil.

Figure 2. Topsoil storage site at the Unidade de Pesquisa e Inovação em Campos Rupestres Ferruginosos da Gerdau, Ouro Branco Municipality, Minas Gerais State, Brazil.

12 famílias (tabela 1). As famílias com maior riqueza de espécies foram Asteraceae (12 espécies), Poaceae (sete), Cyperaceae e Malvaceae (quatro), Convolvulaceae (três), Euphorbiaceae, Fabaceae, Malpighiaceae e Rubiaceae (duas). Apenas duas famílias, Poaceae e Rubiaceae, somaram $65 \%$ do total de indivíduos amostrados, sendo a família Poaceae a mais importante na comunidade. Os gêneros mais ricos em espécies foram Sida L. (três espécies), Borreria G. 


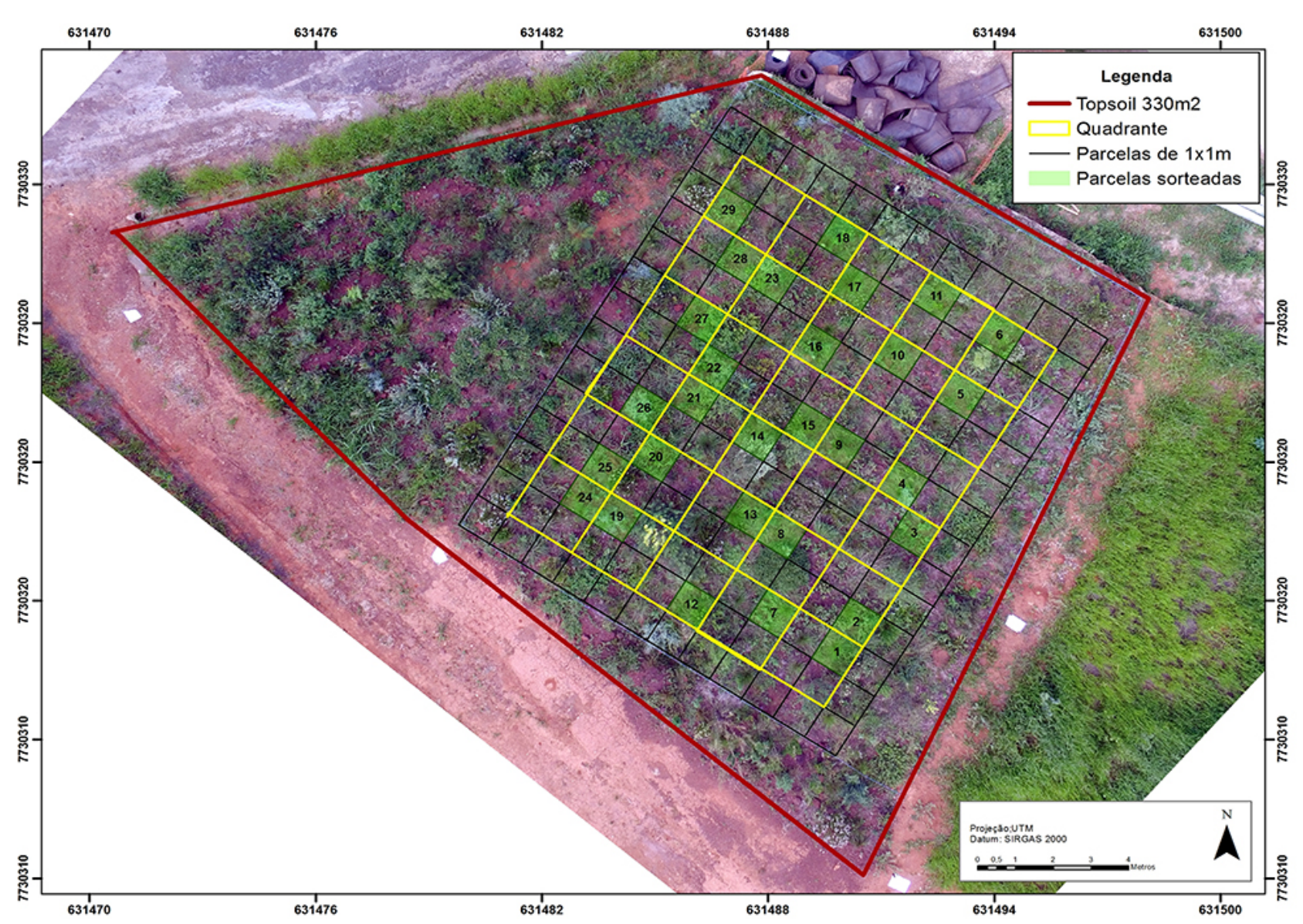

Figura 3. Esquema demonstrativo das parcelas plotadas no topsoil e analisadas nesse estudo, município de Ouro Branco, MG, Brasil.

Figure 3. Schematics of the plots sampled from the analyzed topsoil, Ouro Branco Municipality, Minas Gerais State, Brazil.

Mey., Bulbostylis Kunth., Chromolaena DC. e Eragrostis Wolf (duas) (tabela 1).

As espécies com maior IVI foram Eragrostis polytricha Nees (17,64), Borreria capitata (Ruiz \& Pav.) DC. $(12,85)$, Axonopus siccus (Nees) Kuhlm. (9,20); Bulbostylis junciformis (Kunth.) C.B. Clarke (9,11); Symphyopappus brasiliensis (Gardner) R.M. King \& H. Rob. $(6,46)$ e Urochloa brizantha (Hochst. ex A. Rich.) R.D. Webster $(6,38)$. Os dados de DR foram mais expressivos em $B$. capitata $(25,58)$, E. polytricha $(18,14)$, B. junciformis $(9,53)$, A. siccus $(5,58)$ e $U$. brizantha $(5,35)$. As espécies com maior FR foram E. polytricha $(13,27), B$. capitata $(9,00)$, A. siccus $(9,00), B$. junciformis $(8,53)$ e $S$. brasiliensis $(7,58)$. Os maiores valores de $\mathrm{CR}$ estão associados às seguintes espécies: E. polytricha $(21,5)$, A siccus $(13,02)$, B. junciformis $(9,26)$, S. brasiliensis $(7,14)$ e Borreria $\mathrm{sp}$. $(5,01)$. Todos esses dados podem ser observados na tabela 1 .

A respeito das gramíneas presentes neste levantamento florístico, seis dentre as sete catalogadas são nativas. Dentre essas, pode-se destacar Eragrostis polytricha, Axonopus siccus, e Schizachyrium microstachyum (Desv. ex Ham.) Roseng., B.R. Arrill. \& Izag. A primeira espécie foi a que

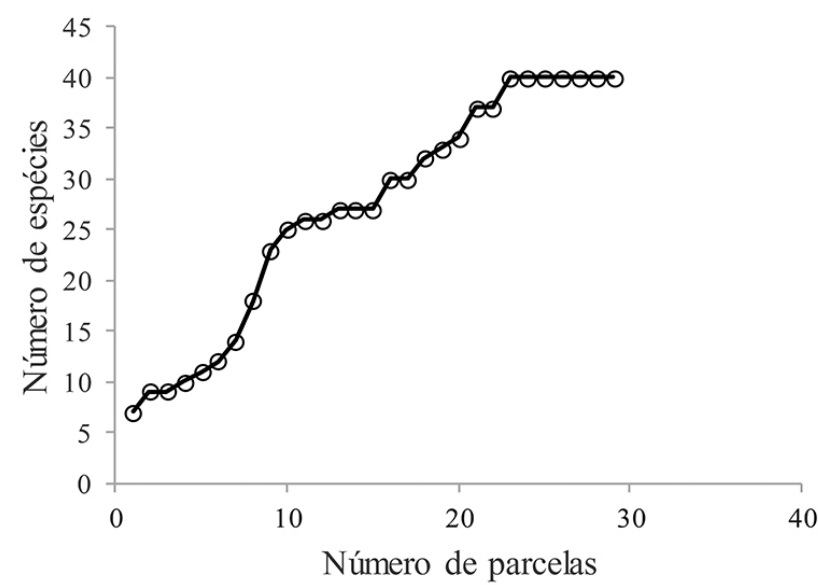

Figura 4. Curva do coletor com as espécies amostradas a partir do desenvolvimento do banco de sementes do topsoil avaliado.

Figure 4. Collector's curve of species sampled upon development of the topsoil seed bank.

apresentou maior IVI além de ser uma das principais também em relação à avaliação de DR (maior valor dentre as espécies 
Tabela 1. Parâmetros fitossociológicos das espécies amostradas em topsoil ferruginoso, Ouro Branco, MG, Brasil. N: Número de indivíduos; NP: Número de parcelas em que a espécie ocorre; DA: Densidade Absoluta; DR: Densidade Relativa; FA: Frequência Absoluta; FR: Frequência Relativa; CA: Cobertura Absoluta; CR: Cobertura Relativa; IVI: Índice de Valor de Importância; *: Espécies com maior IVI.

Table 1. Phytosociological parameters of species sampled from a ferruginous topsoil at Ouro Branco municipality, Minas Gerais State, Brazil. N: Number of individuals; NP: Number of plots in which the species occurs; AD: Absolute Density; RD: Relative Density; AF: Absolute Frequency; RF: Relative Frequency; AC: Absolute Cover; RC: Relative Cover; IV: Importance Value; *: Species with highest IV's.

\begin{tabular}{|c|c|c|c|c|c|c|c|c|c|}
\hline Família/ Espécie & $\mathrm{N}$ & NP & $\mathrm{DA}$ & DR & FA & FR & $\mathrm{CA}$ & $\mathrm{CR}$ & IVI \\
\hline \multicolumn{10}{|l|}{ Amaranthaceae } \\
\hline Pfaffia gnaphaloides (L. f.) Mart. & 4 & 2 & 13,79 & 0,93 & 6,9 & 0,95 & 5 & 0,19 & 0,69 \\
\hline \multicolumn{10}{|l|}{ Asteraceae } \\
\hline Achyrocline satureioides (Lam.) DC. & 6 & 3 & 20,69 & 1,40 & 10,34 & 1,42 & 67,50 & 2,06 & 1,81 \\
\hline $\begin{array}{l}\text { Ayapana amygdalina (Lam.) R.M. King \& } \\
\text { H. Rob. }\end{array}$ & 1 & 1 & 3,45 & 0,23 & 3,45 & 0,47 & 0,01 & 0,00 & 0,24 \\
\hline Baccharis dracunculifolia $\mathrm{DC}$. & 9 & 9 & 31,03 & 2,09 & 31,03 & 4,27 & 107,50 & 4,15 & 3,50 \\
\hline $\begin{array}{l}\text { Chromolaena multiflosculosa (DC.) R.M. } \\
\text { King \& H. Rob. }\end{array}$ & 4 & 4 & 13,79 & 0,93 & 13,79 & 1,90 & 35,00 & 1,35 & 1,39 \\
\hline $\begin{array}{l}\text { Chromolaena pedalis (Sch. Bip. ex Baker) } \\
\text { R.M. King \& H. Rob. }\end{array}$ & 1 & 1 & 3,45 & 0,23 & 3,45 & 0,47 & 2,50 & 0,10 & 0,27 \\
\hline $\begin{array}{l}\text { Chromolaena squalida (DC.) R.M. King } \\
\text { \& H. Rob. }\end{array}$ & 1 & 1 & 3,45 & 0,23 & 3,45 & 0,47 & 62,50 & 2,41 & 1,04 \\
\hline Emilia fosbergii Nicolson & 1 & 1 & 3,45 & 0,23 & 3,45 & 0,47 & 0,01 & 0,00 & 0,24 \\
\hline Indeterminada 1 & 1 & 1 & 3,45 & 0,23 & 3,45 & 0,47 & 15,00 & 0,58 & 0,43 \\
\hline Pterocaulon virgatum (L.) DC. & 2 & 2 & 6,90 & 0,47 & 6,90 & 0,95 & 2,51 & 0,10 & 0,50 \\
\hline Stenophalium chionaeum (DC.) Anderb. & 1 & 1 & 3,45 & 0,23 & 3,45 & 0,47 & 2,50 & 0,10 & 0,27 \\
\hline Stevia urticifolia Thunb. & 1 & 1 & 3,45 & 0,23 & 3,45 & 0,47 & 15,00 & 0,58 & 0,43 \\
\hline $\begin{array}{l}\text { Symphyopappus brasiliensis (Gardner) } \\
\text { R.M. King \& H. Rob. * }\end{array}$ & 20 & 16 & 68,97 & 4,65 & 55,17 & 7,58 & 185,01 & 7,14 & 6,46 \\
\hline \multicolumn{10}{|l|}{ Convolvulaceae } \\
\hline Evolvulus macroblepharis Mart. & 3 & 3 & 10,34 & 0,7 & 10,34 & 1,42 & 32,50 & 1,25 & 1,12 \\
\hline Ipomoea procumbens Mart. ex Choisy & 1 & 1 & 3,45 & 0,23 & 3,45 & 0,47 & 0,01 & 0,00 & 0,24 \\
\hline Jacquemontia linarioides Meisn. & 4 & 4 & 13,79 & 0,93 & 13,79 & 1,90 & 7,51 & 0,29 & 1,04 \\
\hline \multicolumn{10}{|l|}{ Cyperaceae } \\
\hline $\begin{array}{l}\text { Bulbostylis capillaris (L.) Kunth ex C.B. } \\
\text { Clarke }\end{array}$ & 13 & 7 & 44,83 & 3,02 & 24,14 & 3,32 & 17,50 & 0,67 & 2,34 \\
\hline $\begin{array}{l}\text { Bulbostylis junciformis (Kunth) C.B. } \\
\text { Clarke* }\end{array}$ & 41 & 18 & 141,38 & 9,53 & 62,07 & 8,53 & 240,00 & 9,26 & 9,11 \\
\hline $\begin{array}{l}\text { Bulbostylis sphaerocephala (Boeckeler) } \\
\text { Lindm. }\end{array}$ & 1 & 1 & 3,45 & 0,23 & 3,45 & 0,47 & 0,01 & 0,00 & 0,24 \\
\hline Cyperus aggregatus (Willd.) Endl. & 2 & 1 & 6,90 & 0,47 & 3,45 & 0,47 & 2,50 & 0,10 & 0,35 \\
\hline \multicolumn{10}{|l|}{ Euphorbiaceae } \\
\hline Croton antisyphiliticus Mart. & 1 & 1 & 3,45 & 0,23 & 3,45 & 0,47 & 15,00 & 0,58 & 0,43 \\
\hline Microstachys daphnoides (Mart.) Müll. Arg. & 3 & 3 & 10,34 & 0,70 & 10,34 & 1,42 & 42,50 & 1,64 & $\begin{array}{c}1,25 \\
\text { contint }\end{array}$ \\
\hline
\end{tabular}


Tabela 1 (continuação)

\begin{tabular}{|c|c|c|c|c|c|c|c|c|c|}
\hline Família/ Espécie & $\mathrm{N}$ & NP & DA & DR & FA & FR & $\mathrm{CA}$ & $\mathrm{CR}$ & IVI \\
\hline \multicolumn{10}{|l|}{ Fabaceae } \\
\hline Dalbergia miscolobium Benth. & 2 & 2 & 6,90 & 0,47 & 6,90 & 0,95 & 0,02 & 0,00 & 0,47 \\
\hline Vigna sp. & 5 & 5 & 17,24 & 1,16 & 17,24 & 2,37 & 50,00 & 1,93 & 1,82 \\
\hline \multicolumn{10}{|l|}{ Lamiaceae } \\
\hline Hyptis lutescens Pohl ex Benth. & 2 & 1 & 6,90 & 0,47 & 3,45 & 0,47 & 2,10 & 0,10 & 0,35 \\
\hline \multicolumn{10}{|l|}{ Malpighiaceae } \\
\hline Byrsonima variabilis A. Juss. & 1 & 1 & 3,45 & 0,23 & 3,45 & 0,47 & 2,50 & 0,10 & 0,27 \\
\hline Peixotoa tomentosa A. Juss. & 2 & 2 & 6,90 & 0,47 & 6,90 & 0,95 & 30,00 & 1,16 & 0,86 \\
\hline \multicolumn{10}{|l|}{ Malvaceae } \\
\hline $\begin{array}{l}\text { Peltaea polymorpha (A. St.-Hil.) Krapov. } \\
\text { \& Cristóbal }\end{array}$ & 1 & 1 & 3,45 & 0,23 & 3,45 & 0,47 & 15,00 & 0,58 & 0,43 \\
\hline Sida glaziovii K. Schum. & 1 & 1 & 3,45 & 0,23 & 3,45 & 0,47 & 2,50 & 0,10 & 0,27 \\
\hline Sida rufescens A. St.-Hil. & 10 & 4 & 34,48 & 2,33 & 13,79 & 1,90 & 95,00 & 3,66 & 2,63 \\
\hline Sida tuberculata R.E. Fr. & 2 & 2 & 6,90 & 0,47 & 6,90 & 0,95 & 5,00 & 0,19 & 0,54 \\
\hline \multicolumn{10}{|l|}{ Poaceae } \\
\hline Axonopus siccus (Nees) Kuhlm. * & 24 & 19 & 82,76 & 5,58 & 65,52 & 9,00 & 337,50 & 13,02 & 9,20 \\
\hline Chloris aff. ciliata $\mathrm{Sw}$. & 3 & 2 & 10,34 & 0,70 & 6,90 & 0,95 & 17,50 & 0,67 & 0,77 \\
\hline Echinolaena inflexa (Poir.) Chase & 6 & 5 & 20,69 & 1,40 & 17,24 & 2,37 & 25,00 & 0,96 & 1,58 \\
\hline Eragrostis leucosticta Nees ex Döll & 5 & 4 & 17,24 & 1,16 & 13,79 & 1,90 & 2,53 & 0,10 & 1,05 \\
\hline Eragrostis polytricha Nees $*$ & 78 & 28 & 268,97 & 18,14 & 96,55 & 13,27 & 557,50 & 21,50 & 17,64 \\
\hline $\begin{array}{l}\text { Schizachyrium microstachyum (Desv. ex } \\
\text { Ham.) Roseng., B.R. Arrill. \& Izag. }\end{array}$ & 11 & 10 & 37,93 & 2,56 & 34,48 & 4,74 & 110,00 & 4,24 & 3,85 \\
\hline $\begin{array}{l}\text { Urochloa brizantha (Hochst. ex A. Rich.) } \\
\text { R.D. Webster* }\end{array}$ & 23 & 12 & 79,31 & 5,35 & 41,38 & 5,69 & 210,00 & 8,10 & 6,38 \\
\hline \multicolumn{10}{|l|}{ Rubiaceae } \\
\hline Borreria capitata (Ruiz \& Pav.) DC. * & 110 & 19 & 379,31 & 25,58 & 65,52 & 9,00 & 102,71 & 3,96 & 12,85 \\
\hline Borreria sp. & 20 & 8 & 68,97 & 4,65 & 27,59 & 3,79 & 130,00 & 5,01 & 4,49 \\
\hline $\begin{array}{l}\text { Mitracarpus polygonifolius (A. St.-Hil.) } \\
\text { R.M. Salas \& E.B. Souza }\end{array}$ & 2 & 2 & 6,90 & 0,47 & 6,90 & 0,95 & 37,51 & 1,45 & 0,95 \\
\hline \multicolumn{10}{|l|}{ Turneraceae } \\
\hline Turnera oblongifolia Cambess. & 1 & 1 & 3,45 & 0,23 & 3,45 & 0,47 & 2,50 & 0,10 & 0,27 \\
\hline
\end{tabular}

amostradas), FR (segundo maior valor) e CR (maior valor). A única gramínea exótica amostrada foi Urochloa brizantha (tabela 1).

\section{Discussão}

As famílias de maior riqueza no presente estudo estão entre as mais importantes nos Campos Rupestres (Giulietti et al. 1987, Peron 1989, Brandão \& Gavilanes 1990, Brandão et al. 1991, Pirani et al. 1994, Pirani et al. 2003, Menezes \& Giulietti 2000, Zappi et al. 2003, Mendonça 2006, Viana
\& Lombardi 2007, Jacobi et al. 2007, Jacobi \& Carmo 2008, Rapini et al. 2008). Estudos realizados a partir da regeneração de topsoil ferruginoso proveniente da mina de Capão Xavier (Rezende 2010) e da mina da Alegria (Santos 2010), ambas localizadas no Quadrilátero Ferrífero (QF), também apontaram as famílias Fabaceae, Poaceae, Asteraceae, Rubiaceae e Euphorbiaceae como de maior riqueza de espécies.

Em um âmbito geral, áreas de Campo Rupestre Ferruginoso apresentam Asteraceae, Fabaceae e Poaceae dentre as famílias mais representativas (Jacobi et al. 2007, Mourão \& Stehmann 
2007, Viana \& Lombardi 2007, Mendonça 2013), resultados que corroboram com os achados do presente estudo. Em se tratando de diversidade de espécies, nossos dados apontam Asteraceae como a família de maior riqueza. Provavelmente isso se deve ao fato de que os representantes deste grupo se desenvolvem, preferencialmente, em fisionomias campestres (como os Campos Rupestres) e apresentam uma frequência menor em ambientes florestais (Funk et al. 2005). Nesse mesmo contexto, Poaceae também apresentou posição de destaque no presente estudo, sendo a segunda família melhor ranqueada nesse parâmetro. As gramíneas possuem grande amplitude ecológica e se desenvolvem tanto em áreas florestais quanto campestres (Kawakita et al. 2016), no entanto também são encontradas predominantemente em ambientes abertos (Boldrini et al. 2008).

Os resultados obtidos nessa pesquisa mostram a capacidade do topsoil de reconstituir a cobertura vegetal em um período curto de tempo ( 12 meses) com uma considerável diversidade de espécies (42). Destas, seis foram também encontradas por Dias (2016) em uma análise semelhante, a partir de transposição de topsoil ferruginoso em uma área de mineração de bauxita. A utilização de topsoil na revegetação de áreas degradadas tem fornecido resultados promissores em diferentes regiões do globo (Howard \& Samuel 1979, Burke 2008, Rivera et al. 2012, Hall et al. 2010, Ferreira et al. 2015). Segundo Tibbett (2010) o uso de topsoil para revegetação de áreas degradadas é vantajoso e, por isso, um dos métodos mais recomendáveis para esse fim. Essa prática confere benefícios econômicos principalmente através da redução de custos com a compra de mudas e sementes (Rocha 2017).

Rocha (2017), em seu experimento, observou que em um período de 12 meses de recrutamento de topsoil houve uma evolução pedogenética associada à degradação da couraça ferruginosa e uma minimização das perdas por lixiviação em função da comunidade vegetal que se constituiu através do desenvolvimento do banco de sementes. Nesse sentido, é importante destacar que a comunidade formada apresenta uma maior similaridade ao ambiente natural (Conceição \& Pirani 2016). O estabelecimento dessas plantas no topsoil pode promover a liberação de íons $\mathrm{H}^{+}$juntamente com substâncias orgânicas, materiais esses que compõem a formação de quelatos os quais permitem que alguns nutrientes sejam absorvidos pelas raízes com maior facilidade (Larcher 2000).

De acordo com Jacobi et al. (2008), em projetos de reabilitação de áreas degradadas, dispor de variedade de espécies é importante e um dos critérios de escolha pode ser a representatividade de suas famílias tanto em número de espécies quanto em abundância de indivíduos, por indicar o sucesso do táxon em um ambiente particular. Desta forma, os resultados do presente estudo mostram que das espécies presentes no banco de sementes, três gramíneas merecem destaque: Eragrostis polytricha, Axonopus siccus e Schizachyrium microstachyum. Essas espécies são comuns em áreas de Campo Rupestre Ferruginoso e também já foram catalogadas em estudos fitossociológicos realizadas no $\mathrm{QF}$
(Santos 2010, Vincent 2004, Viana \& Lombardi 2007). Urochloa brizantha, única gramínea exótica registrada, se estabeleceu na área possivelmente pela contaminação do topsoil durante o seu transporte ou por meio de chuva de sementes das espécies presentes no entorno do material depositado.

Eragrostis polytricha apresentou o maior IVI entre as espécies amostradas, índice também de destaque em A. siccus e $S$. microstachyum. Em se tratando de espécies potenciais para a utilização em programas de revegetação, o índice de cobertura apresenta grande importância por ser um bom indicador da biomassa da população (Mueller-Dombois \& Ellenberg 1974). A biomassa reflete a performance da população na competição pelos fluxos de matéria e energia na comunidade (Pillar 1996).

Eragrostis polytricha contêm 3-5 flósculos férteis, os quais, na maioria das vezes, resultam em um número igual de sementes. Após a frutificação, as espiguetas podem ser colocadas para germinar sem a necessidade de uma separação individual das sementes, o que pode culminar com a germinação de 3-5 sementes simultaneamente (Saraiva et al. em preparação). Esse processo confere o estabelecimento de um número maior de perfilhos por touceira, fato que provavelmente fornece um aumento na cobertura e no incremento de biomassa nessa espécie (Pereira et al. 2019). Axonopus Siccus e S. microstachyum se reproduzem através de sementes (Rocha \& Secco 2004; Welker \& LonghiWagner 2012). No entanto, em observação pessoal realizada em campo com A. siccus, foi possível perceber que muitas de suas espiguetas são chochas e inviáveis. Assim, uma investigação mais apurada sobre a capacidade dessa espécie investir em desenvolvimento vegetativo é necessária.

Eragrostis polytricha distribui-se pelos Estados Unidos, México, Mesoamérica, América do Sul (exceto Peru, Equador e Suriname) (Boechat \& Longhi-Wagner 2000). Axonopus siccus é registrado para a Argentina, Bolívia, Paraguai, Uruguai e Brasil (Giraldo-Cañas 2010; Flora do Brasil 2020 em construção). Schizachyrium microstachyum distribui-se do México até a Argentina (Peichoto 2010), inclusive no Brasil onde é registrada em quase todos os Estados (Flora do Brasil 2020 em construção). Essas espécies possuem ampla distribuição, ocorrendo não apenas nos Campos Rupestres Ferruginosos, permitindo-nos inferir que possuem mecanismos adaptativos para se estabelecerem em diferentes ambientes, sendo este um importante indicador para o seu uso em áreas degradadas.

Dados provenientes de estudos florísticos conduzidos em Campos Rupestres Ferruginosos podem subsidiar futuros programas de revegetação. No entanto, poucos têm direcionado esforços para avaliar o potencial de gramíneas nativas na utilização em programas de revegetação de taludes em áreas mineradas (Jacob et al. 2008). A maioria dos estudos nessa vertente contemplam espécies exóticas, deixando uma lacuna no conhecimento acerca das espécies nativas. Buisson et al. (2018) mencionam que há uma limitação nos estudos com gramíneas tropicais nativas para esse fim, enquanto 
que nas regiões temperadas esses trabalhos já se encontram em um patamar mais avançado.

As três gramíneas nativas aqui destacadas podem apresentar um potencial interessante para utilização em programas de revegetação. Contudo, E. polytricha por apresentar conjuntamente uma produção em grande quantidade de sementes viáveis em suas panículas (Nees 1829), se desenvolver rapidamente após a semeadura (Obs. Pess.) e possuir considerável área de cobertura, parece ser a mais indicada para esse fim. É importante destacar que a grande produção de sementes viáveis nessa espécie favorece o processo reprodutivo e facilita o aumento de suas populações. Além disso, E. polytricha apresenta grande potencial como pioneira e de acordo com Martins (1996) gramíneas pioneiras desempenham um importante papel no aumento da matéria orgânica do solo e na estabilização do substrato. Uma ilustração da espécie pode ser observada na figura 5 .

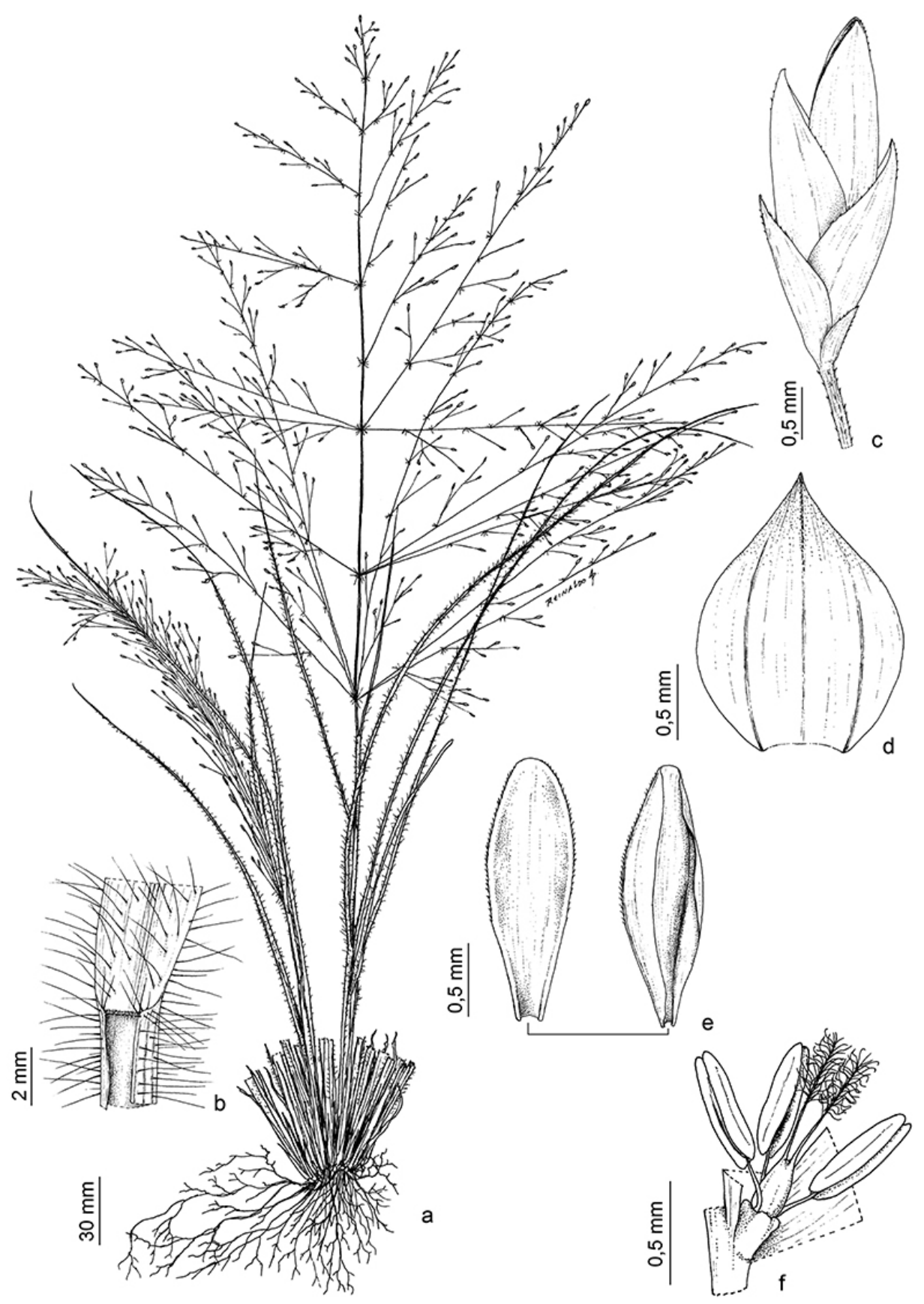

Figura 5. Principais caracteres morfológicos de Eragrotis polytricha Nees. a. Hábito. b. Região entre a lâmina e a bainha foliar. c. Espigueta. d. Lema. e. Pálea. f. Gineceu e androceu. Ilustração: Reinaldo A. Pinto.

Figure 5. Major morphological characters of Eragrotis polytricha Nees. a. Habit. b. Region between the leaf blade and sheath. c. Spikelet. d. Lemma. e. Palea. f. Gynoecium and androecium. Illustration: Reinaldo A. Pinto. 
Apesar deste estudo mostrar que Eragrostis polytricha apresenta potencial para utilização na revegetação de áreas degradadas, ainda se faz necessário a realização de estudos que objetivem compreender mais sobre as condições que essa espécie requer para o processo de germinação em substrato e consequentemente para o desenvolvimento de mudas. Posteriormente, testes em campo serão essenciais para averiguar como será seu comportamento em taludes provenientes de atividade de mineração. A partir de então, será possível realizar uma avaliação sobre o seu potencial para revegetar áreas dessa natureza.

\section{Agradecimentos}

Os autores agradecem à Unidade de Pesquisa e Inovação em Campos Rupestres Ferruginosos da Gerdau, em especial ao gestor Francisco de Assis Lafetá Couto; ao botânico Pablo Meyer, pela contribuição nas identificações das espécies; ao Reinaldo Antônio Pinto, pela confecção da ilustração e ao Mayco S. Werllen-Sousa, pela confecção do mapa. Os autores também agradecem à Universidade Federal de Lavras (UFLA) e à Universidade Federal de Viçosa (UFV), pelo suporte técnico prestado.

\section{Literatura citada}

APG IV (The Angiosperm Phylogeny Group). 2016. An update of the Angiosperm Group classification for the orders and families of flowering plants: APG IV. Botanical Journal of the Linnean Society 181: 1-20.

Araújo, F.S., Martins, S.V., Meira-Neto, J.A.A., Lani, J.L. \& Pires, I.E. 2005. Florística da vegetação arbustivo-arbórea colonizadora de uma área degradada por mineração de caulim, em Brás Pires, MG. Revista Sociedade de Investigações Florestais 29: 983-992.

Braun-Blanquet, J. 1979. Phytosociology. Basis for the study of plant communities. Blume, Madrid.

Brindle, F.A. 2003. Use of native vegetation and biostimulants for controlling soil erosion on steep terrain. Transportation Research Record 1819: 203-209.

Boechat, S.C. \& Longhi-Wagner, H.M. 2000. Padrões de distribuição geográfica dos táxons brasileiros de Eragrostis (Poaceae, Chloridoideae). Revista Brasileira de Botânica 23:177-194.

Boldrini, I.I., Longhi-Wagner, H.M. \& Boechat, S.C. 2008. Morfologia e taxonomia de gramíneas sul-riograndenses. EDUFRGS, Porto Alegre.

Brandão, M. \& Gavilanes, M.L. 1990. Mais uma contribuição para o conhecimento da cadeia do espinhaço em minas gerais, Serra da piedade - II. Daphne 1: 26-43.

Brandão, M., Gavilanes, M.L, Buendia, J.P.L., Macedo, J.F. \& Cunha, L.H.S. 1991. Contribuição para o conhecimento da Cadeia do Espinhaço em Minas Gerais (Serra de Itabirito) - III. Daphne 1: 39-41.
Buisson, E., Le Stradic, S., Silveira, F.A.O., Durigan, G., Overbeck, G.E., Fidelis, A., Fernandes, G.W., Bond, W.J., Hermann, J.M., Mahy, G., Alvarado, S.T., Zaloumis, N.P. \& Veldman, J.W. 2018. Resilience and restoration of tropical and subtropical grasslands, savannas, and grassy woodlands. Biological Reviews doi: $10.1111 /$ brv. 12470

Burke, A. 2008. The effect of topsoil treatment on the recovery of rocky plain and outcrop plant communities in Namibia. Journal of Arid Environments 72: 1531-1536.

Conceicão, A.A. \& Pirani, J.R. 2016. Succession on the Rocky Outcrop Vegetation: A Rupestrian Grassland Scheme. In: Fernandes, G.W. (ed.). Ecology and Conservation of Mountaintop Grasslands in Brazil. Springer International Publishing, Cham, pp.181-206.

Dias, D.D. 2016. Recrutamento e estabelecimento de plantas após transposição de topsoil para área degradada pela mineração de bauxita. Dissertação de Mestrado, Universidade Federal de Ouro Preto, Ouro Preto.

DNPM (Departamento Nacional de Produção Mineral). 2016. Anuário Mineral Brasileiro: Principais Substâncias Metálicas Brasília, Brasil. Disponível em http://www. dnpm.gov.br/dnpm/publicacoes/serie-estatisticas-eeconomia-mineral/anuario-mineral/anuario-mineralbrasileiro/anuario-mineral-brasileiro-2016-metalicos (acesso em 05-I-2018).

Ferreira, M.C., Walter, B.M.T. \& Vieira, D.L.M. 2015. Topsoil translocation for Brazilian savanna restoration: propagation of herbs, shrubs, and trees. Restoration Ecology 23: 723-728.

Flora do Brasil. 2020. Under Construction. Poaceae. Jardim Botânico do Rio de Janeiro. Disponível em http:// floradobrasil.jbrj.gov.br/reflora/floradobrasil/FB13214 (acesso em 31-X-2019).

Funk, V.A., Bayer, R.J., Keeley, S., Chan, R., Watson, L., Gemeinholzer, B., Schilling, E., Panero, J.L., Baldwin, B.G., Garcia-Jacas, N., Susanna, A. \& Jansen, R.K. 2005. Everywhere but Antarctica: using a supertree to understand the diversity and distribution of the Compositae. Biologiske Skrifter 55: 343-374.

Giraldo-Cañas, D. 2010. Novedades taxonómicas en Axonopus (Poaceae, Panicoideae, Paniceae) para Brasil. Rodriguésia 61: 137-142.

Giulietti, A.M., Menezes, N.L., Pirani, J.R., Meguro, M. \& Wanderley, M.G.L. 1987. Flora da Serra do Cipó, Minas Gerais: caracterização e lista de espécies. Boletim de Botânica da Universidade de São Paulo 9: 1-151.

Gyssels, G. \& Poesen, J. 2003. The importance of plant root characteristics in controlling concentrated flow erosion rates. Earth Surface Processes and Landforms 28: 371-384.

Hall, S.L., Barton, C.D. \& Baskin, C.C. 2010. Topsoil seed bank of an Oak-Hickory forest in eastern Kentucky as a restoration tool on surface mines. Restoration Ecology 18: 834-842. 
Howard, G.S. \& Samuel, M.J. 1979.The value of freshstripped topsoil as a source of useful plants for surface mine revegetation. Journal of Range Management 32: 76-77.

IBRAM (Instituto Brasileiro de Mineração). 2015. Informações sobre a economia mineral brasileira. Disponível em http://www.ibram.org.br/ sites/1300/1382/00005836.pdf (acesso em 05-I-2017).

Jacobi, C.M., Carmo, F.F., Vincent, R.C. \& Stehmann, J.R. 2007. Plant communities on ironstone outcrops - a diverse and endangered Brazilian ecosystem. Biodiversity and Conservation 16: 2185-2200.

Jacobi, C.M. \& Carmo, F.F. 2008. Diversidade dos Campos Rupestres Ferruginoso no Quadrilátero Ferrífero, MG. Megadiversidade 4:24-32.

Jacobi, C.M., Carmo, F.F. \& Vincent, R.C. 2008. Estudo fitossociológico de uma comunidade vegetal sobre canga como subsídio para a reabilitação de áreas mineradas no Quadrilátero Ferrífero, MG. Revista Árvore 32: 345-353.

Kawakita, K., Rodrigues, R.S. \& Filgueiras, T.S. 2016. Poaceae em uma planície de inundação no Brasil: listagem florística e novas ocorrências. Hoehnea 43: 203-216.

Larcher, W. 2000. Ecofisiologia Vegetal. RIMA, São Carlos.

Marques, T.E.D. 2011. Uso de gramíneas em consórcio com leguminosas para recuperação de voçorocas. Dissertação de Mestrado, Universidade Federal de Ouro Preto, Ouro Preto.

Martins, C.R. 2001.Recuperação de uma área pela mineração de cascalho com o uso de gramíneas nativas. Revista Árvore 25: 157-166.

Martins, C.R. 1996. Revegetação com gramíneas de uma área degradada no Parque Nacional de Brasília, DF, Brasil. Dissertação de Mestrado, Universidade de Brasília, Brasília.

Martins, F.R \& Santos, F.A.M. 1999. Técnicas usuais de estimativa da biodiversidade. Holos 1: 236-267.

Mendonca, M.P. 2006. Coleta e cultivo das espécies vegetais dos Campos Ferruginosos: Mina de minério de ferro Capão Xavier, Nova Lima - MG. Relatório de Atividades. Fundação Zoobotânica de Belo Horizonte, Belo Horizonte.

Mendonça, M.P.O. 2013. Resgate da Flora da Canga. Valor Natural, Belo Horizonte.

Menezes, N.L. \& Giulietti, A.M. 2000. Campos Rupestres. In: Mendonça, M.P., Lins, L.V. (Orgs.). Lista Vermelha das Espécies Ameaçadas de Extinção da Flora de Minas Gerais. Fundação Biodiversitas e Fundação Zoobotânica de Belo Horizonte, Belo Horizonte, pp. 65-73.

Mourão, A. \& Stehmann, J.R. 2007. Levantamento da Flora do Campo Rupestre Sobre Canga Hematítica Couraçada Remanescente na Mina do Brucutu, Barão de Cocais, MG. Rodriguésia 58: 775-786.
Mueller-Dombois, D. \& Ellenberg, H. 1974. Aims and Methods of Vegetation Ecology. John Wiley \& Sons, New York.

Nees, C.G. 1829. Gramineae. In: Martius, C.F.P, Eichler, A.W. \& Urban, I. (eds). Flora Brasiliensis seu Enumeratio Plantarum in Brasilia tam sua sponte quam accedente cultura provenientium, quas in itinere auspiciis Maximiliani Josephi I. Bavariae Regis annis 1817-1820 peracto collegit, partim descripsit; alias a Maximiliano Seren. Principe Widensi, sellovio aliisque advectas addidit. Sumptibus J.G. Cottae, Stuttgartiae, pp. 1-608.

Peichoto, M.C. 2010. Revisión taxonómica de las especies del género Schizachyrium (Poaceae: Andropogoneae) de Sudamérica. Candollea 65: 301-346.

Pereira, L.D., Mauro, L.B., Sucharski, Y.L., Morais, E., Silva, R. \& Coelho, C. 2019. Efeitos de diferentes substratos na emergência e crescimento de plântulas de Eragrostis polytricha a partir de sementes e espiguetas. In: IV Seminário de Iniciação Científica e Extensão do IFMG - Ouro Branco. Instituto Federal de Minas Gerais, Ouro Branco.

Pérez, E.M. \& Santiago, E.T. 2001. Dinámica estacional del banco de semillasen una sabanaenlos Lhanos CentroOrientales de Venezuela. Biotropica 33: 435-446.

Peron, M.V. 1989. Listagem preliminar da Flora Fanerogâmica dos Campos Rupestres do Parque Estadual do Itacolomi - Ouro Preto/Mariana, MG. Rodriguésia 67: 63-69.

Pillar, V.D. 1996. Descrição de comunidades vegetais. Universidade Federal do Rio Grande do Sul, Departamento de Botânica. Disponível em http://ecoqua. ecologia.ufrgs.br (acesso em 10-I-2018).

Pirani, J.R., Giulietti, A.M., Mello-Silva, R. \& Meguro, M. 1994. Checklist and patterns of geographic distribution of the vegetation of Serra do Ambrósio, Minas Gerais, Brazil. Revista Brasileira de Botânica 17:133-147.

Pirani, J.R., Mello-Silva, R. \& Giulietti, A.M. 2003. Flora de Grão Mogol, Minas Gerais, Brasil. Boletim de Botânica da Universidade de São Paulo 21: 1-24.

Rapini, A., Ribeiro, P.L., Lambert, S. \& Pirani, J.R. 2008. A flora dos Campos Rupestres da Cadeia do Espinhaço. Megadiversidade 4: 15-23.

Rezende, L.A.L. 2010. Reabilitação de campos ferruginosos degradados pela atividade minerária no Quadrilátero Ferrífero. Dissertação de Mestrado, Universidade Federal de Viçosa, Viçosa.

Rivera, D., Peco, B. \& Jáuregui, B.M. 2012. The fate of herbaceous seeds during topsoil stockpiling: Restoration potential of seed banks. Ecological Engineering 44: 94-101.

Rocha, F.C.G. 2017. Evolução de características físicas e químicas de topsoil de campo ferruginoso aplicado em projeto de recuperação de área degradada pela mineração. Dissertação de Mestrado, Universidade Federal de Ouro Preto, Ouro Preto. 
Rocha, A.S. \& Secco, R. 2004. Contribuição à taxonomia de Axonopus P.Beauv. (Poaceae) no estado do Pará, Brasil. Acta Botanica Brasilica 18: 295-304.

Salomão, R.P. 2015. Restauração Florestal de Precisão: dinâmica e espécies estruturantes - Evolução de áreas restauradas em uma unidade de conservação na Amazônia; Porto Trombetas, Pará. Novas Edições Acadêmicas, Saarbücken.

Santos, L.M. 2010. Restauração de campos ferruginosos mediante resgate de flora e uso de topsoil no Quadrilátero Ferrífero, Minas Gerais. Tese de Doutorado, Universidade Federal de Minas Gerais, Belo Horizonte.

Silva, M.F.F., Secco, R.S. \& Lobo, M.G. 1996. Aspectos ecológicos da vegetação rupestre da Serra dos Carajás, estado do Pará, Brasil. Acta Amazônica 26: 17-44.

Tibbett, M. 2010. Large-scale mine site restoration of Australian eucalypt forests after bauxite mining: soil management and ecosystem development. In: Batty, I. \& Hallberg, K. (eds). Ecology of industrial pollution. Cambridge University Press, Cambridge, pp.309-326.
Viana, P.L. \& Lombardi, J.A. 2007. Florística e caracterização dos Campos Rupestres sobre canga na Serra da Calçada, Minas Gerais, Brasil. Rodriguésia 58: 159-177.

Vincent, R.C. 2004. Florística, fitossociologia e relações entre a vegetação e o solo em área de Campos Ferruginosos no Quadrilátero Ferrífero, Minas Gerais. Tese de Doutorado, Universidade de São Paulo, São Paulo.

Welker, C.A.D. \& Longhi-Wagner, H.M. 2012. Sinopse do gênero Schizachyrium Nees (Poaceae - Andropogoneae) no estado do Rio Grande do Sul, Brasil. Iheringia 67: 199-223.

Zappi, D.C., Lucas, E., Stannard, B.L., Lughadha, E., Pirani, J.R., Queiroz, L.P., Atkins, S., Hind, N., Giulietti, A.M., Harley, R.M., Mayo, S.J. \& Carvalho, A.M. 2003. Lista de plantas vasculares de Catolés, Chapada Diamantina, Bahia, Brasil. Boletim de Botânica da Universidade de São Paulo 21: 345398. 